\title{
Quantifying differences in hepatic uptake of the liver specific contrast agents Gd-EOB-DTPA and Gd-BOPTA: a pilot study
}

Olof Dahlqvist Leinhard, Nils Dahlström, Johan Kihlberg, Per Sandström, Torkel Brismar,

Örjan Smedby and Peter Lundberg

\section{Linköping University Post Print}

N.B.: When citing this work, cite the original article.

The original publication is available at www.springerlink.com:

Olof Dahlqvist Leinhard, Nils Dahlström, Johan Kihlberg, Per Sandström, Torkel Brismar, Örjan Smedby and Peter Lundberg, Quantifying differences in hepatic uptake of the liver specific contrast agents Gd-EOB-DTPA and Gd-BOPTA: a pilot study, 2011, European Radiology, (22), 3, 642-653.

http://dx.doi.org/10.1007/s00330-011-2302-4

Copyright: Springer Verlag (Germany)

http://www.springerlink.com/

Postprint available at: Linköping University Electronic Press http://urn.kb.se/resolve?urn=urn:nbn:se:liu:diva-73624 
Quantifying Differences in Hepatic Uptake of the Liver Specific Contrast Agents Gd-EOB-DTPA and Gd-BOPTA: a Pilot Study

Authors, affiliations:

Dahlqvist Leinhard 01,3, Dahlström N ${ }^{1,2}$, Kihlberg J1,4, Sandström P6, Brismar $\mathrm{TB}^{5}$, Smedby $\mathrm{Ö}^{1,2}$, and Lundberg $\mathrm{P}^{1,2,3}$

1. Center for Medical Image Science and Visualization (CMIV), Linköping University, Linköping, Sweden.

2. Division of Radiology, Department of Medical and Health Sciences (IMH), Faculty of Health Sciences, Linköping University, Dept of Radiology, Center for Medical Imaging, UHL, County Council of Östergötland, Linköping, Sweden.

3. Division of Radiation Physics, Department of Medical and Health Sciences, Faculty of Health Sciences, Linköping University, Dept of Radiation Physics, UHL, County Council of Östergötland, Linköping, Sweden.

4. Dept of Cardiovascular Medicine (IMH), Linköping University, Dept of Radiology, Center for Medical Imaging, UHL, County Council of Östergötland, Linköping, Sweden.

5. Dept of Clinical Science, Intervention and Technology at Karolinska Institutet, Division of Medical Imaging and Technology, Dept of Radiology, Karolinska University Hospital in Huddinge, Stockholm, Sweden.

6. Division of Surgery and Clinical Oncology, Department of Clinical and Experimental Medicine (IKE), Faculty of Health Sciences, Linköping University, Department of Surgery in Östergötland, County Council of Östergötland, Linköping, Sweden. 


\title{
Quantifying Differences in Hepatic Uptake of the Liver Specific Contrast Agents Gd-EOB-DTPA and Gd-BOPTA: a Pilot Study
}

\begin{abstract}
Objectives: To develop and evaluate a procedure for quantifying the hepatocyte-specific uptake of Gd-BOPTA and Gd-EOB-DTPA using Dynamic Contrast-Enhanced (DCE) MRI.

Methods: Ten healthy volunteers were prospectively recruited and 21 patients with suspected hepatobiliary disease were retrospectively evaluated. All subjects were examined with DCE-MRI using $0.025 \mathrm{mmol} / \mathrm{kg}$ of Gd-EOB-DTPA. The healthy volunteers underwent an additional examination using $0.05 \mathrm{mmol} / \mathrm{kg}$ of Gd-BOPTA. The signal intensities (SI) of liver and spleen parenchyma were obtained from unenhanced and enhanced acquisitions. Using pharmacokinetic models of the liver and spleen and an SI rescaling procedure, a hepatic uptake rate, $K_{\text {Hep }}$, estimate was derived. $K_{\text {Hep }}$ values for Gd-EOB-DTPA were then studied in relation to those for GdBOPTA and to a clinical classification of the patient's hepatobiliary dysfunction.

Results: $K_{\text {Hep }}$ estimated using Gd-EOB-DTPA showed a significant Pearson correlation with $K_{\text {Hep }}$ estimated using Gd-BOPTA $(r=0.64 ; p<0.05)$ in healthy subjects. Patients with impaired hepatobiliary function had significantly lower $K_{\mathrm{Hep}}$ than patients with normal hepatobiliary function $\left(K_{\mathrm{Hep}}=0.09 \pm 0.05 \mathrm{~min}^{-1}\right.$ versus $\left.K_{\mathrm{Hep}}=0.24 \pm 0.10 \mathrm{~min}^{-1} ; \mathrm{P}<0.01\right)$.

Conclusions: A new procedure for quantifying the hepatocyte-specific uptake of $T_{1}$-enhancing contrast agent was demonstrated and used to show that impaired hepatobiliary function severely influences the hepatic uptake of Gd-EOB-DTPA.
\end{abstract}

Keywords: Gadolinium ethoxybenzyl diethylenetriaminepentaacetic acid, Gadobenate Dimeglumine, Dynamic Contrast-Enhanced MRI, Pharmacokinetics, Liver 
Key points

1. The liver uptake of contrast agents may be measured with standard clinical MRI.

2. Calculation of liver contrast agent uptake is improved by considering splenic uptake.

3. Liver function affects the uptake of the liver specific contrast agent Gd-EOB-DTPA.

4. Hepatic uptake of two contrast agents (Gd-EOB-DTPA, Gd-BOPTA) is correlated in healthy individuals.

5. This method can be useful for determining liver function, e.g. before hepatic surgery 


\section{Introduction}

Dynamic contrast-enhanced magnetic resonance imaging (DCE-MRI) methods with both gadoxetate (Gd-EOB-DTPA; Primovist $\left.{ }^{(}\right)$Bayer Schering Pharma, Berlin, Germany) and gadobenate dimeglumine (Gd-BOPTA; MultiHance ${ }^{\circledR}$ Bracco Imaging, Milan, Italy) have been proposed as promising methods for the characterisation of liver function and staging of liver fibrosis $[1,2]$. There is a major pharmacokinetic difference regarding the elimination of the two contrast agents: Gd-EOB-DTPA has a higher hepatobiliary excretion, 50\%, compared with 2-4\% for Gd-BOPTA [3].

Based on investigations in a rat model, Tsuda et al. have shown that characterisation of both the early and the late uptake of Gd-EOB-DTPA enables separation of rats with non-alcoholic fatty liver disease (NAFLD) from rats with non-alcoholic steatohepatitis (NASH) [4]. In another study by Planchamp et al., a relationship between the pharmacokinetic parameters of Gd-BOPTA uptake in rat liver was shown to correlate negatively with the degree of liver fibrosis [5]. Recently, a retrospective study on patients by Motosugi et al. described a relationship between liver 'signal intensity' (SI) in the late phases after Gd-EOB-DTPA injection and liver function measured with Indocyanine Green (ICG) clearance and Child-Pugh class [6].

The transfer of analysis methods developed in rat models to studies in humans is difficult, as the pharmacokinetics of Gd-EOB-DTPA and Gd-BOPTA are slower in humans. The complete time course of the accumulation and excretion of contrast agents is too long to be observed in a standard clinical setting. Still, it is feasible to analyse the contrast agent accumulation in the hepatobiliary phase, as has been described by Motosugi et al. and Yamada et al., who used a semi-quantitative approach relating the liver SI to the splenic SI $[7,8]$.

An important problem in contrast-enhanced MRI is the fact that SI is defined on an arbitrary scale which can neither be compared between repeated examinations of the same patient, nor between different patients. Differences in radiofrequency (RF) coil loading, $B_{1}$-field heterogeneity, repetition times $\left(T_{\mathrm{R}}\right)$, longitudinal relaxation time $\left(T_{1}\right)$, transverse relaxation time $\left(T_{2}\right.$ or $\left.T_{2}^{*}\right)$ and patient/respiratory motion all influence the observed SI [9]. Although techniques exist that are capable of producing quantitative images of the $T_{1}$ relaxation [10], a number of critical image quality parameters, such as spatial or temporal resolution, volume coverage and compensation for patient motion, are sub-optimal for a satisfactory clinical characterisation of focal lesions. However, by careful analysis of the signal intensities in $T_{1}$-weighted gradient echo (GRE) imaging, it is possible to calculate a time series of sufficiently accurate relaxation rate values that are intrinsically proportional to contrast agent concentration although the possible influence of disease- or treatment-induced alterations in native tissue $T_{1}$ should be considered 
[11]. Such quantitatively derived estimates are useful and even necessary for reproducible and robust evaluation of function and morphology, which could be applied in, e.g., preoperative liver function evaluation for planning of segmental liver resections or in treatment evaluation.

The aim of the present work was to develop a procedure for quantifying the hepatocytespecific contrast agent uptake during late time phases using standard imaging protocols. This procedure was evaluated in healthy volunteers using both Gd-EOB-DTPA and Gd-BOPTA, in order to investigate whether the uptake of Gd-BOPTA correlated with the hepatic uptake of GdEOB-DTPA. The hepatocyte-specific uptake was also evaluated in patients in order to investigate whether impaired hepatobiliary function influences the hepatic uptake of Gd-EOB-DTPA. 


\section{Materials and Methods}

\section{Subjects}

The study comprised two different categories of subjects: healthy volunteers examined using both Gd-EOB-DTPA and Gd-BOPTA, and patients examined using only Gd-EOB-DTPA.

The healthy volunteer group consisted of five women (mean age: 25 years, range: 22-33 years) and five men (mean age: 25 years, range: 23-27 years). The patient group, including all patients who had been examined with Gd-EOB-DTPA-enhanced MRI of the liver for suspected hepatobiliary disease from December 2006 to August 2008, was evaluated retrospectively. One examination was found to be incomplete because of technical failure and was for this reason excluded from the analysis. Thus, 21 patients -13 men and 8 women aged 21-81 years (mean: 55 years) - were included.

For all patients, serum bilirubin, and other laboratory tests were retrieved to enable calculation of Child-Pugh [12] and 'Model for End-Stage Liver Disease' (MELD) [13] scores.

The clinical grading of the hepatobiliary function of every patient at the time of the MRI examination, using the electronic patient records but without knowledge of MRI analyses, was performed retrospectively by a liver surgeon in three separate groups according to Table 1 . The study protocol was approved by the regional ethics committees in Stockholm and Linköping, Sweden.

\section{Contrast Agents}

All healthy volunteers were examined on two occasions at least 3 weeks apart using two different liver-specific contrast agents, $0.025 \mathrm{mmol} / \mathrm{kg}$ of Gd-EOB-DTPA (Primovist ${ }^{\circledR} 0.25 \mathrm{mmol} / \mathrm{ml}$, Bayer Schering Pharma, Berlin, Germany), and $0.05 \mathrm{mmol} / \mathrm{kg}$ of Gd-BOPTA (MultiHance ${ }^{\circledR} 0.5$ $\mathrm{mmol} / \mathrm{ml}$; Bracco Imaging, Milan, Italy). The bolus was injected manually at $1 \mathrm{~mL} / \mathrm{s}$, immediately followed by an equal amount of saline. The arterial phase data acquisition was started $15-20 \mathrm{~s}$ post-injection, and the venous phase started approximately $60 \mathrm{~s}$ post-injection.

The patients were examined in accordance with our standard clinical liver MRI protocol. $0.025 \mathrm{mmol} / \mathrm{kg}$ of Gd-EOB-DTPA was administered intravenously at a rate of $1 \mathrm{~mL} / \mathrm{s}$ using a power injector (Medrad Spectris Solaris, Pittsburgh, PA, USA), and immediately followed by an equal amount of saline. The timing of the arterial phase was determined by real-time bolus monitoring at the level of the upper abdominal aorta. Delayed acquisitions of the hepatobiliary phase were obtained in both groups, as specified in Table 2 . 


\section{Data Acquisition}

All examinations were performed on a $1.5 \mathrm{~T}$ magnetic resonance system (Achieva, Philips Medical Systems, Best, The Netherlands), using a phased-array body coil. The examinations were performed after at least $7 \mathrm{~h}$ of fasting. The study protocol to examine the healthy subjects contained one unenhanced and six enhanced acquisitions of an axial single breath-hold, fatsaturated, $T_{1}$-weighted, 3D GRE sequence. The clinical protocol contained a total of five enhanced acquisitions. Pulse sequence details are given in Table 2.

\section{Image Analysis}

Signal intensity curves were obtained using the averages from seven regions of interest (ROIs) located in the liver and three ROIs in the spleen. The liver ROIs were distributed in both the left and right lobes, and placed in parenchymal areas without any large vessels, or focal lesions, see Fig. 1. Particular care was taken to preserve the correct scaling of the signal in the original images in order to enable the analysis and comparison of signal measurements from different images in the time series.

\section{Normalisation of SI}

The SI time series $(S I(t))$ from each individual ROI was then normalised by dividing it by the unenhanced signal intensity $(S I(0))$. The signal normalisation removed constant background effects on the SI that occurred throughout the time series resulting from differences in image sensitivity, proton density, transverse relaxation and fatty infiltration. Thus, the normalised signal intensity, $S$, is given by:

$$
S(t)=\frac{S I(t)}{S I(0)}
$$

\section{Conversion of SI Values to Relaxation Rate}

The normalised SI values were then recalculated to relaxation rate values, assuming fixed unenhanced or intrinsic $T_{1}$ values, $T_{10}=T_{1}(0)$. These were $586 \mathrm{~ms}$ for the liver and $1057 \mathrm{~ms}$ for the spleen ROIs [14]. Correction was also made for non-linear effects from the chosen $T_{\mathrm{R}}$, and the flip angle $(\alpha)$ in this procedure. The signal intensity in a gradient echo sequence [15] follows approximately,

$$
S I(t)=\xi \cdot \sin (\alpha) \cdot \frac{1-E(t)}{1-\cos (\alpha) \cdot E(t)}
$$

where $\xi$ is a constant describing the sensitivity of the MRI, and $E(t)$ is equal to 


$$
E(t)=e^{-\frac{T_{R}}{T_{1}(t)}}
$$

Then,

$$
S(t)=\frac{1-E(t)}{1-\cos (\alpha) \cdot E(t)} \cdot \frac{1-\cos (\alpha) \cdot E(0)}{1-E(0)} .
$$

Re-arrangement of this equation leads to the following non-linear expression for $R_{1}(t)=1 / T_{1}(t)$ :

$$
\frac{1}{T_{1}(t)}=-\frac{1}{T_{R}} \cdot\left(\ln \left(S(t) \cdot \frac{1-E(0)}{1-\cos (\alpha) \cdot E(0)}-1\right)-\ln \left(\cos (\alpha) \cdot S(t) \cdot \frac{1-E(0)}{1-\cos (\alpha) \cdot E(0)}-1\right)\right) .
$$

A step-by-step illustration of the process is provided in Fig. 2, where the effects of different intrinsic $T_{1}$ and of noise are also demonstrated.

There are two important reasons to incorporate such correction procedures in the analysis of signal intensities in quantitative dynamic contrast-enhanced studies. First, the slope in the SI response to contrast medium concentration is a function of the intrinsic tissue $T_{1}$ before injection of a contrast agent. Second, there is also a non-linear response of the SI for $T_{1}$ values that are short in relation to the $T_{\mathrm{R}}$ and flip angle.

\section{Calculation of Contrast Agent Concentration}

After recalculation of the SI to $R_{1}$ values, a linear relation [16] that depends on the contrast agent concentration $(\mathrm{C})$ in the tissue was assumed as follows:

$$
C \cdot r_{1}=\Delta R_{1}=\left\{R_{1}(t)-R_{1}(0)\right\}
$$

where $r_{1}$ is the relaxivity of the contrast agent in situ in the tissue and $\Delta R_{1}$ the induced increase in the relaxation rate. The assumed linear relationship between contrast agent concentration in the tissue and $\Delta R_{1}$ allowed us to calculate a 'dose normalised' $\Delta R_{1}$ by multiplying by $1 / 2$ the estimated $\Delta R_{1}$ in the examinations of healthy volunteers using Gd-BOPTA, as the injected dose of Gd-BOPTA was twice the injected dose of Gd-EOB-DTPA.

\section{Pharmacokinetic Analysis}

The pharmacokinetic analysis of the time series was based on a two-compartment model of the liver and spleen (Fig. 3). The splenic tissue was represented by one compartment consisting of splenic blood and 'extracellular extravascular space' (EES) which was exposed to the blood contrast agent concentration, and one intracellular compartment that was inaccessible to the contrast agent. The liver tissue was similarly described using one blood and EES compartment 
exposed to the blood contrast agent concentration and one hepatobiliary compartment, accessible to the contrast agent via its transport over the basolateral membrane of the hepatocytes. The model did not distinguish between hepatocytic intracellular contrast agent and contrast agent transported into the biliary ductules.

The volume fractions of the combined blood and EES compartments to the total tissue water content in the liver $\left(\varphi_{\text {Liver }}\right)$ and spleen $\left(\varphi_{\text {Spleen }}\right)$ were used to adjust for the different signal contributions from the different compartments. In this model the total increase in $R_{1}$ in the liver $\left(\Delta R_{1 \text { Liver }}\right)$ is given by

$$
\Delta R_{1 \text { Liver }}=\left(1-\varphi_{\text {Liver }}\right) \Delta R_{1 \text { Hepatobiliary }}+\varphi_{\text {Liver }} \Delta R_{1 \text { Blood\&EES }}
$$

The corresponding $R_{1}$ increase in the spleen $\left(\Delta R_{1 \text { Spleen }}\right)$, assuming no contribution from the intracellular splenic compartment, was related to the $R_{1}$ increase in the blood and EES compartment ( $\left.\Delta R_{1 \text { Blood\&EES }}\right)$ via the following relation

$$
\Delta R_{1 \text { Spleen }}=\varphi_{\text {Spleen }} \Delta R_{1 \text { Blood\&EES }} .
$$

From these equations, the increase in hepatobiliary $R_{1}\left(\Delta R_{1 \text { Hepatobiliary }}\right)$ was defined as follows:

$$
\Delta R_{1 \text { Hepatobilary }}=\left(\Delta R_{1 \text { Liver }}-\frac{\varphi_{\text {Liver }}}{\varphi_{\text {Spleen }}} \cdot \Delta R_{1 \text { Spleen }}\right) /\left(1-\varphi_{\text {Liver }}\right)
$$

The conversion of $\Delta R_{1 \text { Hepatobiliary }}$ to contrast agent concentration was not straightforward because of uncertainty regarding the exact values of $\varphi_{\text {Liver }}$ and $\varphi_{\text {Spleen }}$, and the tissue-specific relaxivity in hepatocytes and bile. Furthermore, the comparison of $\Delta R_{1 \text { Hepatobiliary }}$ between different subjects was sensitive to a number of different factors that are not easily corrected, such as the patientspecific liver volume, renal function and the injected dose in comparison with the blood plasma volume. To minimise the influence from such inter-examination effects, the relative hepatobiliary accumulation of contrast agent $\left(F_{\text {Hep }}\right.$ ) was calculated by dividing $\Delta R_{1 \text { Hepatobiliary }}$ with $\Delta R_{1 \text { Blood\&EES }}$ obtained from the measurements in the spleen, thus:

$$
\begin{aligned}
& F_{\text {Hep }}=\frac{\Delta R_{1 \text { Hepatobilary }}}{\Delta R_{1 \text { Blood\&EES }}}= \\
& =\frac{\varphi_{\text {Spleen }}}{\left(1-\varphi_{\text {Liver }}\right)} \cdot\left(\Delta R_{1 \text { Liver }}-\frac{\varphi_{\text {Liver }}}{\varphi_{\text {Spleen }}} \cdot \Delta R_{1 \text { Spleen }}\right) / \Delta R_{\text {1Spleen }}= \\
& =\frac{\varphi_{\text {Spleen }}}{\left(1-\varphi_{\text {Liver }}\right)} \cdot\left(\frac{\Delta R_{1 L i v e r}}{\Delta R_{\text {ISpleen }}}-\frac{\varphi_{\text {Liver }}}{\varphi_{\text {Spleen }}}\right) .
\end{aligned}
$$


The exact value of the volume fraction factors is not very critical in the model, but may be approximated according to the values of a 'standard human subject' using $\varphi_{\text {Liver }}=0.23$ and $\varphi_{\text {Spleen }}=0.3[17]$, and therefore:

$$
F_{\text {Hep }}=0.39 \cdot\left(\frac{\Delta R_{1 \text { Liver }}}{\Delta R_{1 \text { Spleen }}}-0.77\right) .
$$

After inspection of the dynamics of $F_{\text {Hep }}$ following the arterial phase measurement, it was proposed that a linear increase in $F_{\text {Hep }}$ during the hepatobiliary phases occurred in the subjects. Therefore a least squares regression analysis was used to obtain the slope $\left(K_{\mathrm{Hep}}\right)$ from the following relation:

$$
F_{\text {Hер }}=K_{\text {Hер }} \cdot t \text {. }
$$

Here $t$ is the contrast agent exposure time. The major advantage obtained by the introduction of such a procedure (Eq. 11) was that all observations in the time series after the arterial phase could be used in order to improve the signal-to-noise ratio. In late time phases using Gd-EOB-DTPA, the estimation of $F_{\text {Hep }}$ was more sensitive to noise in the estimated $C_{\text {Spleen }}$ because of blood concentrations near the detection limit. Therefore, only observations of up to 20 min were used in the fitting procedures of the individual data series.

\section{Other Measures of Late Hepatobiliary Uptake and Blood Plasma Clearance}

Unprocessed SI measurements from liver and spleen ROIs were used to calculate the quantitative liver-spleen contrast (LSC) ratio, according to the description in Motosugi et al. [7] at 10 (LSC10) and 20 (LSC20) min after contrast agent administration,

$$
L S C 10=\frac{S I_{\text {Liver }}(10)}{S I_{\text {Spleen }}(10)} \text { and } L S C 20=\frac{S I_{\text {Liver }}(20)}{S I_{\text {Spleen }}(20)}
$$

In the context of SI normalisation being used in the pharmacokinetic model, it is reasonable to attempt normalisation of the liver and spleen SI values to form a modified LSC ratio, here named the normalised liver-to-spleen contrast ratio, LSC_N, according to the following expressions:

$$
L S C_{-} N=\frac{S_{\text {Liver }}}{S_{\text {Spleen }}} \text {. }
$$

The splenic blood clearance rate (SBC) for each subject was estimated by least squares fitting of the estimated $\Delta R_{1 \text { Spleen }}$ to a monoexponential function, 


$$
\Delta R_{1 \text { Spleen }}(t)=a \cdot e^{-t \cdot S B C},
$$

where $a$ is the amplitude scaling factor and $t$ is the time after contrast agent injection.

\section{Statistics}

Interval variables were described with mean and standard deviation, and ordinal variables using median and range. $K_{\text {Hep }}$, LSC and LSC_N in each healthy subject for both Gd-EOB-DTPA and Gd-BOPTA were compared using pairwise Pearson correlation and also linear regression. The group means of $K_{\mathrm{Hep}}$, bilirubin, LSC and LSC_N representing clinical grades 1-3 in the patient group were compared using a one-way ANOVA. As the Levene test showed unequal variances across groups for almost all tested parameters, post-hoc testing was performed according to Games-Howell using BrightStat [18]. Except for the Games-Howell test, statistical calculations were performed in JMP 6.0 (SAS Institute, Cary, NC, USA) or MATLAB R2009a (The MathWorks, Inc. Massachusetts, USA).

\section{Results}

\section{Hepatobiliary Uptake}

\section{Healthy subjects}

Mean and standard errors of SI, normalised SI and dose normalised $\Delta R_{1}$ in the splenic and liver ROIs for all ten healthy subjects using Gd-EOB-DTPA and Gd-BOPTA are shown in Fig. 4, panel A-C. In panel D the time series of $F_{\text {Hep }}$ is shown together with the ratios of $F_{\text {Hep }}$ determined using Gd-EOB-DTPA and Gd-BOPTA respectively. Calculated uptake rates $K_{\mathrm{Hep}}$, LSC and LSC_N ratios and clearance rates are listed in Table 3. The pairwise correlation between the fitted $K_{\text {Hep }}$ for each subject using Gd-EOB-DTPA and Gd-BOPTA was significant $(\mathrm{P}<0.05)$ with $r=0.64$ (Fig. 5A). The fitted regression slope was (estimate \pm standard error) $7.42 \pm 3.66$, and the fitted intercept was $0.14 \pm 0.12$. When a constrained intercept (equal to zero) was used in the regression the estimated slope was $11.0 \pm 1.65$. Conversely, no strong correlation was observed between the hepatic uptake of Gd-EOB-DTPA and Gd-BOPTA using LSC20 (Fig. 5B).

\section{Patients}

Calculated uptake rates $K_{\text {Hep }}$, LSC and LSC_N ratios, bilirubin values, blood clearance rates, Child-Pugh and MELD scores are reported in Table 4, where diagnoses in clinical grades 1-3 are also listed. In one of the clinical grade 1 patients, it was not possible to obtain the LSC20/LSC_N20. 
The group means of $K_{\mathrm{Hep}}, \mathrm{LSC}_{-} \mathrm{N} 10$ and LSC_N20 of the clinical grades 1-3 were significantly different and pairwise comparisons yielded significant differences between clinical grades 1 and 2, and between 1 and 3, but not between clinical grades 2 and 3 (Table 4). Also for LSC10, LSC20 and bilirubin the group means were significantly different. The Games-Howell test showed significant differences between clinical grades 1 and 3, and between 2 and 3 for both LSC10 and LSC20. For bilirubin, there was a significant difference only between clinical grades 1 and 3 .

\section{Plasma Clearance Rate}

The plasma clearance rate estimated using SBC was significantly higher using Gd-EOB-DTPA than Gd-BOPTA ( $p<0.002$; paired Student's t-test) (Table 3). Among the patients a significantly lower SBC was observed in the clinical grade 3 group compared with the clinical grade 1 group (Table 4).

\section{Discussion}

\section{Quantitative Measurement of the Hepatobiliary Contrast Agent Uptake}

In this work, a method for the quantification of hepatocyte-specific contrast agent kinetics in the human liver was developed based on standard 3D $T_{1}$-weighted GRE images acquired using high spatial resolution and full liver coverage. The results clearly illustrate that quantitative analysis of the signal response of a $T_{1}$-enhancing contrast agent provides greater insight into the underlying contrast agent kinetics. By estimation of $\Delta R_{1}$ using well-known physical relationships, a measure of the contrast agent concentration in the tissue was achieved. The method described here, in which the hepatic contrast agent concentration was correlated to the plasma concentration (as observed in the spleen), enables both quantitative measurements and characterisation of the late hepatic uptake. In contrast to the procedure proposed by Motosugi et al., who also used the splenic tissue as reference tissue [7], the method presented here provides physiologically interpretable values.

The assumption of fixed intrinsic (unenhanced) relaxation values, $T_{10}$, of the tissues is a simplification. The $T_{10}$ of liver used here was $586 \mathrm{~ms}$, reported by de Bazelaire et al. [14], with a standard deviation of $39 \mathrm{~ms}$. Thomsen et al. [19] described a larger variation and they reported that the $T_{1}$ is prolonged in cirrhosis patients. Van Lom et al. [20] also reported more variation, $T_{10}$ $=568 \pm 59 \mathrm{~ms}$ (mean $\pm \mathrm{SD}$ ). The addition of a quantitative $T_{1}$-mapping sequence would provide the means to address interindividual variations as well as intraindividual changes, e.g. due to treatment-induced tissue changes, in order to improve the pharmacokinetic analysis. Furthermore, 
quantitative imaging methods [21] would probably lead to a more finely detailed analysis with respect to errors in flip angles, coil sensitivity and patient motion.

However, while the pulse sequences used in clinical DCE-MRI of the liver are capable of imaging small anatomical structures with a very large field of view, within the time of a single breath-hold, and are insensitive to fatty infiltration, they are not primarily designed for quantitative imaging. We therefore believe that the quantification methodology presented here may represent an important step forward in clinical DCE-MRI.

\section{Characteristics of the Hepatobiliary Uptake}

The hepatic uptake rate $K_{\text {Hep }}$ provided significant separation of patients without hepatobiliary disease from those with hepatobiliary disease, both without and with manifest biliary stasis; there was no significant difference between the two latter groups. Conversely, the serum bilirubin level and the simple liver-to-spleen ratio (LSC) showed a significant difference between the two patient groups with hepatobiliary disease, but no significant separation between these and patients with normal function. We therefore believe that the apparently greater ability of $K_{\text {Hep }}$ and the normalised LSC_N ratios to discriminate between clinical grades 1 and 2 is an indication that the $K_{\text {Hep }}$ estimate has a test response suitable for early detection of disturbed hepatobiliary function. The similar discrimination capability of LSC_N implies that the signal normalisation procedure is the most important factor for removing patient-specific bias in the functional characterisation.

Contrary to mere normalisation as used here to obtain LSC_N, the process to convert SI to contrast agent concentration values adjusts for the (inherently) non-linear influence of important MRI parameters such as $T_{\mathrm{R}}$, native $T_{1}$ and flip angle, for each acquisition. In addition, several image acquisitions in late time phases can be used in calculating $K_{\text {Hep }}$, whereas LSC_N relies on only one time point. Furthermore, improvements in determining native $T_{1}$ will directly be applicable to the calculation of $K_{\mathrm{Hep}}$. In a different context that does not advocate or allow this level of quantitative analysis, the LSC_N is likely a better alternative than the non-normalised, SI-based LSC ratio.

The ratio of hepatobiliary uptake of Gd-EOB-DTPA to that of Gd-BOPTA was estimated through a fitted regression slope, which amounted to an uptake rate approximately 11 times higher for Gd-EOB-DTPA, in agreement with pre-clinical studies [3, 22]. In addition, the plasma clearance rate determined from splenic ROI measurements was higher for Gd-EOB-DTPA, which agrees with its shorter plasma half-life compared with Gd-BOPTA [3, 22].

The lack of accurate non-invasive measures of liver dysfunction was addressed by applying a clinician's grading of the patient hepatobiliary function. A retrospective study by Tajima et al. [23] on differences between patients with chronic liver dysfunction and those with normal liver 
function has some similarities to our patient study in that the patient records formed the basis of patient grouping. Prospective study designs incorporating for example, liver biopsies and ICG clearance tests, can improve the classification of hepatobiliary dysfunction and provide assessment of possible bias by gender or age. Another limitation is the assumption that the blood and EES compartment of the spleen is similar to that of the liver. This introduces sensitivity to splenic disease into the method. The practical significance of this limitation should also be defined in prospective studies.

Lee et al. [24] found in a study on living liver donors that Gd-EOB-DTPA and Gd-BOPTA are equally useful to characterize vascular and biliary variations, at least from a qualitative point of view. This finding is strengthened by the results from the quantitative evaluation model presented here, i.e. the observation that the uptakes of the two contrast agents are correlated. Our interpretation is that the estimated uptake rate of either contrast agent may represent a similar aspect of liver function, possibly indicating a close relationship between the patient-specific characteristics of these contrast agents. From a methodological point of view, the observed correlation and the approximately tenfold ratio between hepatobiliary Gd-EOB-DTPA and GdBOPTA uptake rates measured in healthy subjects suggest that the method was able to accurately estimate the hepatobiliary uptake, despite large differences in blood contrast agent concentration kinetics and administered dose. Future studies will be needed to ascertain whether $K_{\text {Hep }}$ values may be compared between studies differing in equipment, injection and acquisition protocols, a goal that one can hardly expect to reach with the simpler LSC_N measure.

This method enables quantitative assessment of the hepatic uptake of liver specific contrast agents. The clinical relevance of determining the hepatic uptake of Gd-EOB-DTPA and GdBOPTA is not yet proved, but the method is suitable as a combined tool for characterization of regional and global liver function, especially as focal lesions may be characterized within the same examination. Animal investigations have shown promising correlation between hepatobiliary contrast uptake and liver fibrosis $[4,5]$. In agreement with the results by Motosugi et al., the observations in this study indicate that hepatobiliary function can be assessed with our method. The possibility to assess regional contrast agent uptake may also be useful for preoperative characterization and quantification of liver function in patients undergoing hepatic surgery, e.g. segmental hepatectomy. Since the $K_{\mathrm{Hep}}$ estimate is quantitatively derived from local image data, it provides a foundation for various parametric analyses on a segmental or pixel level.

In conclusion, a signal rescaling procedure to correct for the non-linear SI response of T1weighted images acquired using a standard clinical abdominal DCE imaging protocol has been demonstrated. Utilising simple pharmacokinetic modelling, the hepatobiliary uptake of Gd-EOB- 
DTPA and Gd-BOPTA was shown to correlate well in healthy subjects, and the ratio of the hepatobiliary uptake between the two contrast agents was in agreement with preclinical studies. In addition, it was shown that impaired hepatobiliary function influences the hepatic uptake of Gd-EOB-DTPA.

\section{Acknowledgements}

Financial support from the Swedish research council (VR/M 2007-2884), the Medical Research Council of Southeast Sweden (FORSS 12621), and the Linköping University Hospital Research Foundations are gratefully acknowledged. We thank research nurse Annika Hall at the Center for Medical Image Science and Visualization (CMIV) for assistance with volunteer data collection.

\section{References}

Tsuda N, Okada M, Murakami T (2010) New proposal for the staging of nonalcoholic steatohepatitis: evaluation of liver fibrosis on Gd-EOB-DTPA-enhanced MRI. Eur J Radiol, 73(1):137-142.

2 Planchamp C, Pastor CM, Balant L, Becker CD, Terrier F, Gex-Fabry M (2005) Quantification of Gd-BOPTA uptake and biliary excretion from dynamic magnetic resonance imaging in rat livers: model validation with 153Gd-BOPTA. Invest Radiol, 40(11):705-714. doi: 00004424-200511000-00004 [pii]

3 Spinazzi A, Lorusso V, Pirovano G, Kirchin M (1999) Safety, tolerance, biodistribution, and MR imaging enhancement of the liver with gadobenate dimeglumine: results of clinical pharmacologic and pilot imaging studies in nonpatient and patient volunteers. Acad Radiol, 6(5):282-291.

4 Tsuda N, Okada M, Murakami T (2007) Potential of gadolinium-ethoxybenzyl-diethylenetriamine pentaacetic acid (Gd-EOB-DTPA) for differential diagnosis of nonalcoholic steatohepatitis and fatty liver in rats using magnetic resonance imaging. Invest Radiol, 42(4):242-247. doi: 10.1097/01.rli.0000258058.44876.a5 00004424200704000-00007 [pii]

5 Planchamp C, Gex-Fabry M, Becker CD, Pastor CM (2007) Model-based analysis of Gd-BOPTA-induced MR signal intensity changes in cirrhotic rat livers. Invest Radiol, 42(7):513-521. doi: 10.1097/RLI.0b013e318036b450 00004424-200707000-00006 [pii]

6 Motosugi U, Ichikawa T, Sou H, et al. (2009) Liver parenchymal enhancement of hepatocyte-phase images in GdEOB-DTPA-enhanced MR imaging: which biological markers of the liver function affect the enhancement? J Magn Reson Imaging, 30(5):1042-1046. doi: 10.1002/jmri.21956

7 Motosugi U, Ichikawa T, Tominaga L, et al. (2009) Delay before the hepatocyte phase of Gd-EOB-DTPAenhanced MR imaging: is it possible to shorten the examination time? Eur Radiol, 19(11):2623-2629. doi: 10.1007/s00330-009-1467-6

8 Yamada A, Hara T, Li F, et al. (2011) Quantitative Evaluation of Liver Function with Use of Gadoxetate Disodium-enhanced MR Imaging. Radiology. doi: radiol.11100586 [pii] 10.1148/radiol.11100586

9 Jackson A (2004) Analysis of dynamic contrast enhanced MRI. Br J Radiol, 77 Spec No 2:S154-166.

10 Treier R, Steingoetter A, Fried M, Schwizer W, Boesiger P (2007) Optimized and combined T1 and B1 mapping technique for fast and accurate T1 quantification in contrast-enhanced abdominal MRI. Magn Reson Med, 57(3):568-576. doi: 10.1002/mrm.21177

11 Huang W, Wang Y, Panicek DM, Schwartz LH, Koutcher JA (2009) Feasibility of using limited-populationbased average R10 for pharmacokinetic modeling of osteosarcoma dynamic contrast-enhanced magnetic resonance imaging data. Magn Reson Imaging, 27(6):852-858. doi: S0730-725X(09)00035-6 [pii] 10.1016/j.mri.2009.01.020

12 Pugh RN, Murray-Lyon IM, Dawson JL, Pietroni MC, Williams R (1973) Transection of the oesophagus for bleeding oesophageal varices. Br J Surg, 60(8):646-649.

13 Kamath PS, Wiesner RH, Malinchoc M, et al. (2001) A model to predict survival in patients with end-stage liver disease. Hepatology, 33(2):464-470.

14 de Bazelaire CM, Duhamel GD, Rofsky NM, Alsop DC (2004) MR imaging relaxation times of abdominal and pelvic tissues measured in vivo at $3.0 \mathrm{~T}$ : preliminary results. Radiology, 230(3):652-659. doi: 10.1148/radiol.2303021331 230/3/652 [pii]

15 Ernst RR, Anderson WA (1966) Application of fourier transform spectroscopy to magnetic resonance. Review of Scientific Instruments, 37(1):93-102. 
16 Pintaske J, Martirosian P, Graf H, et al. (2006) Relaxivity of Gadopentetate Dimeglumine (Magnevist), Gadobutrol (Gadovist), and Gadobenate Dimeglumine (MultiHance) in human blood plasma at 0.2, 1.5, and 3 Tesla. Invest Radiol, 41(3):213-221. doi: 10.1097/01.rli.0000197668.44926.f7 00004424-200603000-00003 [pii]

17 Levitt DG (2003) The pharmacokinetics of the interstitial space in humans. BMC Clin Pharmacol, 3:3. doi: 10.1186/1472-6904-3-3

18 Stricker D (2008) BrightStat.com: free statistics online. Comput Methods Programs Biomed, 92(1):135-143. doi: S0169-2607(08)00158-2 [pii] 10.1016/j.cmpb.2008.06.010

19 Thomsen C, Christoffersen P, Henriksen O, Juhl E (1990) Prolonged T1 in patients with liver cirrhosis: an in vivo MRI study. Magn Reson Imaging, 8(5):599-604.

20 Van Lom KJ, Brown JJ, Perman WH, Sandstrom JC, Lee JK (1991) Liver imaging at 1.5 tesla: pulse sequence optimization based on improved measurement of tissue relaxation times. Magn Reson Imaging, 9(2):165-171. doi: 0730-725X(91)90006-8 [pii]

21 Warntjes JB, Leinhard OD, West J, Lundberg P (2008) Rapid magnetic resonance quantification on the brain: Optimization for clinical usage. Magn Reson Med, 60(2):320-329. doi: 10.1002/mrm.21635

22 Hamm B, Staks T, Muhler A, et al. (1995) Phase I clinical evaluation of Gd-EOB-DTPA as a hepatobiliary MR contrast agent: safety, pharmacokinetics, and MR imaging. Radiology, 195(3):785-792.

23 Tajima T, Takao H, Akai H, et al. (2010) Relationship between liver function and liver signal intensity in hepatobiliary phase of gadolinium ethoxybenzyl diethylenetriamine pentaacetic acid-enhanced magnetic resonance imaging. J Comput Assist Tomogr, 34(3):362-366. doi: 10.1097/RCT.0b013e3181cd330400004728201005000-00007 [pii]

24 Lee MS, Lee JY, Kim SH, et al. (2011) Gadoxetic acid disodium-enhanced magnetic resonance imaging for biliary and vascular evaluations in preoperative living liver donors: comparison with gadobenate dimeglumineenhanced MRI. J Magn Reson Imaging, 33(1):149-159. doi: 10.1002/jmri.22429

Table 1. Grades 1-3 used in clinical classification, clinical grades 1-3.

\section{Clinical Grade Description}

$1 \quad$ Mild manifestations of, or no hepatobiliary disease, i.e. "normal".

Hepatic or biliary disease processes* likely affecting hepatobiliary function but no major biliary obstruction present.

*e.g. small volume, severe cirrhosis, thromboses, primary sclerosing cholangitis.

Manifest biliary stasis.

Table 2. Parameters for the axial breath-hold fat-saturated $T_{1}$-weighted $3 \mathrm{D}$ gradient echo sequence (THRIVE).

\begin{tabular}{|l||c|c|}
\hline Parameter & Volunteers & Patients \\
\hline TR [ms] & 5.2 & 4.3 \\
\hline TE $[\mathrm{ms}]$ & 2.5 & 2.1 \\
\hline Flip Angle [ $\left.{ }^{\circ}\right]$ & 10 & 10 \\
\hline Slice Thickness (Acquired) [mm] & 3.3 & 4 \\
\hline Reconstructed voxel size [mm] & 1.6 & 2 \\
\hline Acquisition Matrix & $256 / 164$ & $192 / 192$ \\
\hline
\end{tabular}




\begin{tabular}{|l||c|c|}
\hline Percent Phase FOV & 70 & 79 \\
\hline Coil & 4-element body coil & 16-element body coil \\
\hline Acquisition time $[\mathrm{s}]$ & $30-40$ & $20-25$ \\
\hline Sensitivity encoding (SENSE) & No & Yes \\
\hline Reconstruction Diameter & 400 & 395 \\
\hline Acquisition time phases & $\begin{array}{c}\text { Unenhanced, arterial, } \\
\text { portal venous, 10, 20, } \\
30 \text { and } 40 \text { min. }\end{array}$ & $\begin{array}{c}\text { Unenhanced, arterial, } \\
\text { portal venous, } 5,10 \text { and } \\
20 \text { min. }\end{array}$ \\
\hline Reconstructed voxel size $\left[\mathrm{mm}^{3}\right]$ & $1.6 \times 1.6 \times 1.6$ & $1.6 \times 1.6 \times 2.0$ \\
\hline
\end{tabular}

Table 3. Hepatobiliary uptake of Gd-EOB-DTPA and Gd-BOPTA in 10 healthy subjects estimated using the liver-to-spleen contrast ratio at 10 and 20 min post-contrast agent injection (LSC10 and LSC20), the modified LSC ratio obtained after normalisation using the native liver and spleen ROI signal intensities, (LSC_N10, LSC_N20), and $K_{\text {Hep }}$ describing the hepatobiliary specific contrast agent uptake in late time phases. The splenic blood clearance rate (SBC) is also given. $A$ paired t-test showed significant differences between the Gd-EOB-DTPA and the Gd-BOPTA groups for all uptake and clearance parameters. Correlation is reported with significance levels indicated by $*_{p}<0.05$. NS: Not significant.

Pearson P-value Gd-EOB-DTPA Gd-BOPTA correlation correlation

\begin{tabular}{|l||c|c||c|c||}
\hline$K_{\text {Hер }}\left(\mathrm{min}^{-1}\right)$ & $0.37 \pm 0.19$ & $0.03 \pm 0.02$ & 0.64 & $*$ \\
\hline LSC10 & $2.34 \pm 0.27$ & $1.54 \pm 0.22$ & -0.14 & NS \\
\hline LSC20 & $2.66 \pm 0.32$ & $1.79 \pm 0.21$ & -0.07 & NS \\
\hline LSC_N10 & $1.32 \pm 0.11$ & $0.87 \pm 0.06$ & 0.20 & NS \\
\hline LSC_N20 & $1.50 \pm 0.16$ & $0.97 \pm 0.07$ & 0.35 & NS \\
\hline SBC $\left(\mathrm{min}^{-1}\right)$ & $0.08 \pm 0.03$ & $0.05 \pm 0.01$ & 0.58 & NS \\
\hline
\end{tabular}

Table 4. Description of patients' classification according to clinical grade (as defined in Table 1). Diagnoses of clinical grades 1-3 are listed immediately below the table. Values are displayed as means \pm standard deviations, except for the MELD and Child-Pugh Scores which are described 
using median and range. ANOVA and post hoc comparisons of clinical grade 1, 2 and 3 according to the Games-Howell test are shown with significance levels indicated by ${ }^{* * *} p<0.001,{ }^{* *} p<0.01$, $*_{p}<0.05$. NS: Not significant.

\begin{tabular}{|c|c|c|c|c|c|c|c|}
\hline & \multicolumn{3}{|c|}{ Clinical Grade } & \multirow{2}{*}{$\frac{\text { ANOVA }}{F}$} & \multicolumn{3}{|c|}{ Games-Howell } \\
\hline & 1 & 2 & 3 & & 1 vs 2 & 1 vs 3 & 2 vs 3 \\
\hline$N$ & 9 & 5 & 7 & & & & \\
\hline Age & $48 \pm 20$ & $53 \pm 18$ & $66 \pm 12$ & & & & \\
\hline MELD Score & $6[5-10]$ & 12 [9-19] & 15 [12-33] & & & & \\
\hline Child-Pugh Score & $6[5-8]$ & $6[5-8]$ & $6[5-10]$ & & & & \\
\hline P-Bilirubin $(\mu \mathrm{mol} / \mathrm{l})$ & $9 \pm 4$ & $54 \pm 58$ & $153 \pm 94$ & $11^{* *}$ & NS & $*$ & NS \\
\hline$K_{\text {Hep }}$ rate $\left(\min ^{-1}\right)$ & $0.24 \pm 0.1$ & $0.09 \pm 0.05$ & $0.02 \pm 0.02$ & $21 * * *$ & $* *$ & $* * *$ & NS \\
\hline LSC10 Ratio & $1.64 \pm 0.35$ & $1.58 \pm 0.09$ & $1.11 \pm 0.18$ & $9 * *$ & NS & $* *$ & $* * *$ \\
\hline LSC20 Ratio & $1.87 \pm 0.31$ & $1.71 \pm 0.06$ & $0.75 \pm 0.23$ & $16 * * *$ & NS & $* * *$ & $* *$ \\
\hline LSC_N10 Ratio & $1.33 \pm 0.13$ & $1.05 \pm 0.15$ & $0.87 \pm 0.1$ & $27 * * *$ & $*$ & $* * *$ & NS \\
\hline LSC_N20 Ratio & $1.45 \pm 0.16$ & $1.14 \pm 0.15$ & $0.92 \pm 0.11$ & $26 * * *$ & $*$ & $* * *$ & NS \\
\hline SBC Clear. $\left(\mathrm{min}^{-1}\right)$ & $0.08 \pm 0.05$ & $0.04 \pm 0.02$ & $0.04 \pm 0.02$ & $5 *$ & NS & $*$ & NS \\
\hline
\end{tabular}

Clinical Grade 1 patients: gall bladder carcinoma stage 1B, Crohn's disease, ulcerative colitis, moderate (one) and severe (one) liver steatosis, mild primary sclerosing cholangitis (PSC), colon cancer metastases (small, resected), resected solitary hepatocellular carcinoma with no recurrence after 2 years.

Clinical Grade 2 patients: 3 with PSC, 2 of which had cholangiocarcinoma without biliary stasis, 1 patient with recurrent PSC after transplantation and 1 patient with portal vein thrombosis under treatment.

Clinical Grade 3 patients: 3 with bile duct stone(s), 1 with recurring non-patency problems after hepaticojejunostomy, 1 with pancreatic head cancer, with a Klatskin tumour Bismuth $3 A$, and 1 with a gall bladder tumour. 


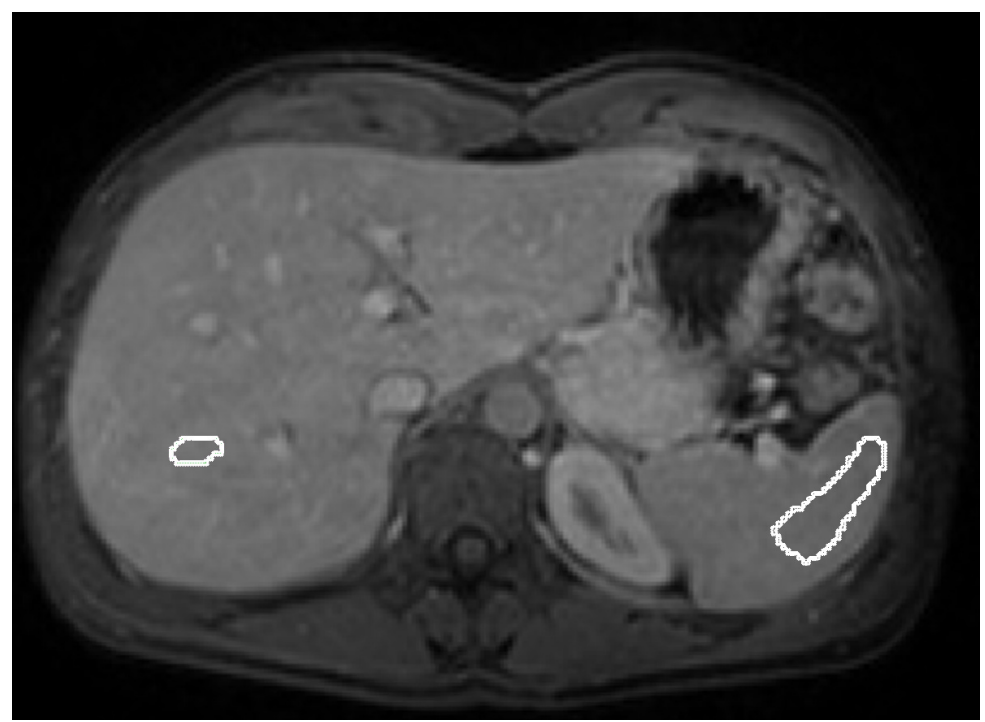

Fig. 1. Region of interest (ROI) samples in the liver and spleen. ROIs were placed in both the left and right lobes of the liver and in several different slices representing both caudal and cranial regions of the liver and spleen. 

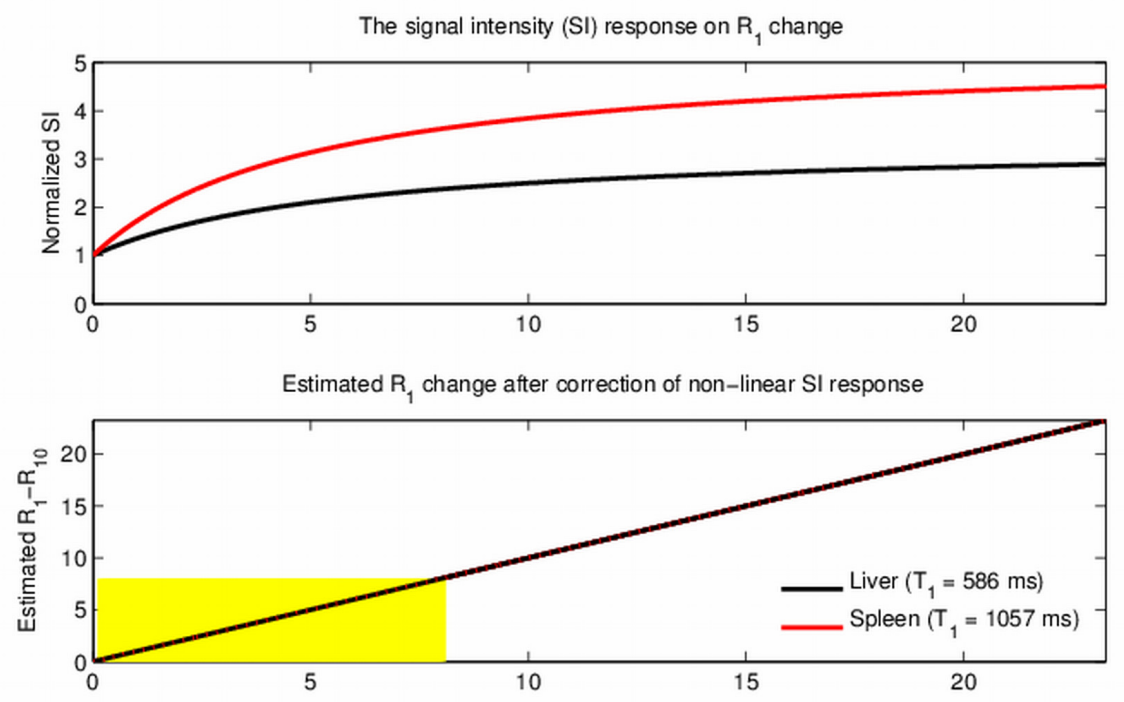

The effect of changing $T_{10}$ with $\pm S D$ as reported by de Bazelaire et al.

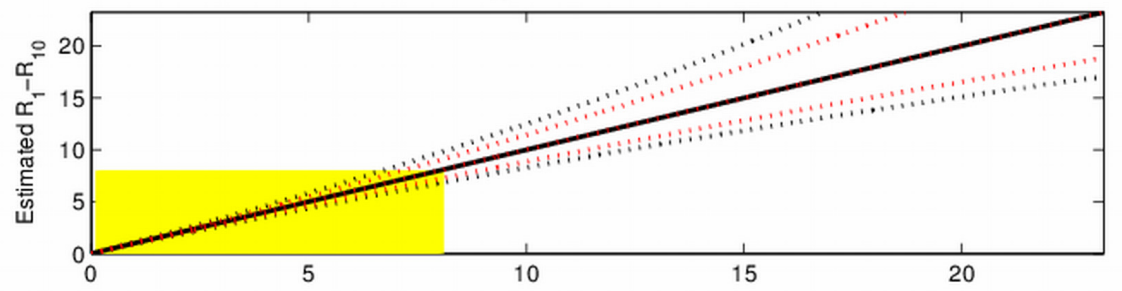

The effect of adding $10 \%$ noise

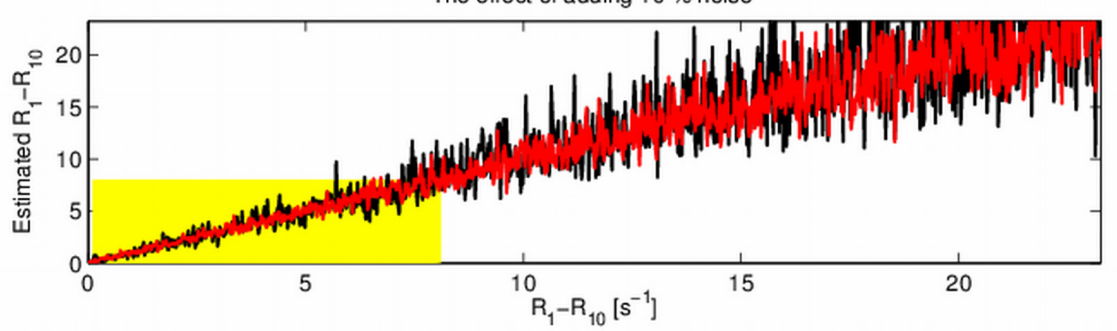

Fig. 2. Modelling of the behaviour of the signal intensity (SI) to relaxation rate $\left(R_{1}\right)$ rescaling procedure. UPPER PANEL: The non-linear SI response according to Eq. 2 on the contrast agent concentration in liver (BLACK line) and splenic (RED line) tissue respectively, for an $R_{1}$ increase from zero to $25 \mathrm{~s}^{-1}$. UPPER MIDDLE PANEL: After application of Eq. 4 the increase in normalised SI was recalculated to an increase in $R_{1}$ values. The yellow area indicates the $R_{1}$ increase observed in this study. LOWER MIDDLE PANEL: The effect of unenhanced $T_{10}$ values deviating from the expected by \pm one standard deviation (SD) observed in healthy individuals by de Bazelaire et al. [14] (39 ms for liver and 42 ms for spleen). LOWER PANEL: the sensitivity of the $R_{1}$ recalculation to noise was investigated by adding Gaussian noise $(10 \%$ of the unenhanced SI) to the normalised SI values. 


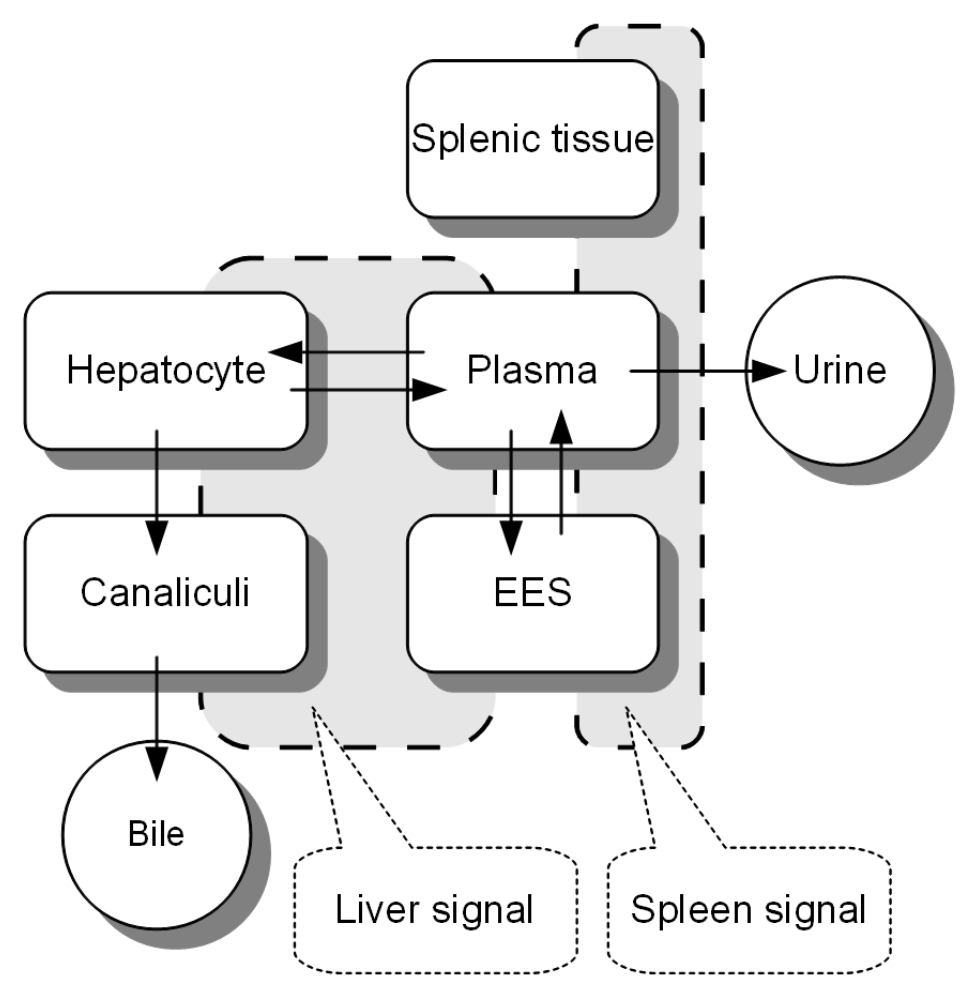

Fig. 3. Simple pharmacokinetic model. The dashed grey boxes represent the liver and the spleen, where the measurement ROIs in the images give the signal intensities. The white boxes represent the compartments of the model, where plasma and the extracellular extravascular space (EES) are part of both the liver and the spleen. "Splenic tissue" represents the structures of the spleen inaccessible to Gd-EOB-DTPA. Arrows show the direction of contrast agent transport between compartments. The excreted contrast agent is collected in bile and urine, represented by white circles. 

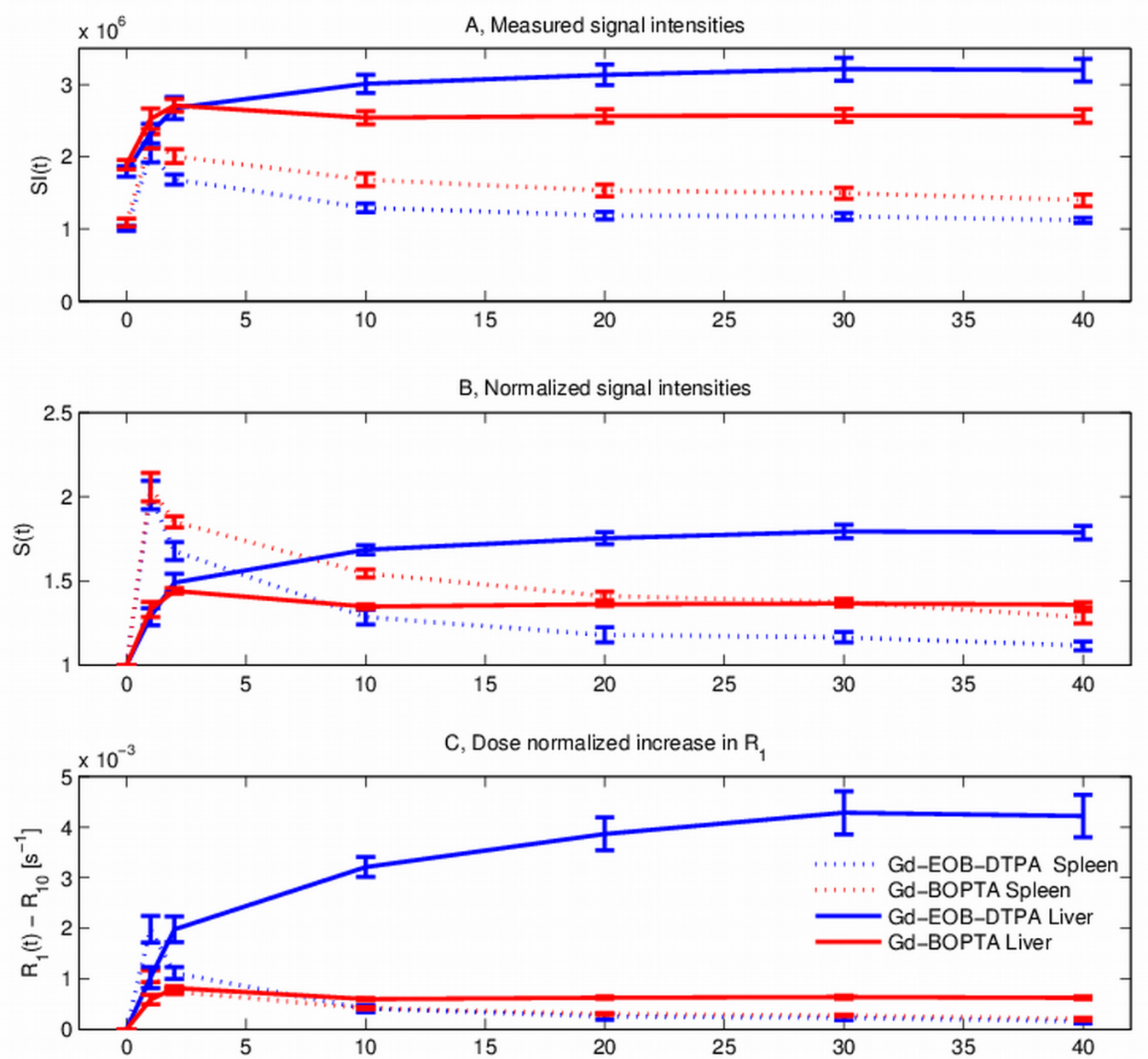

D, Relative hepatobiliary uptake

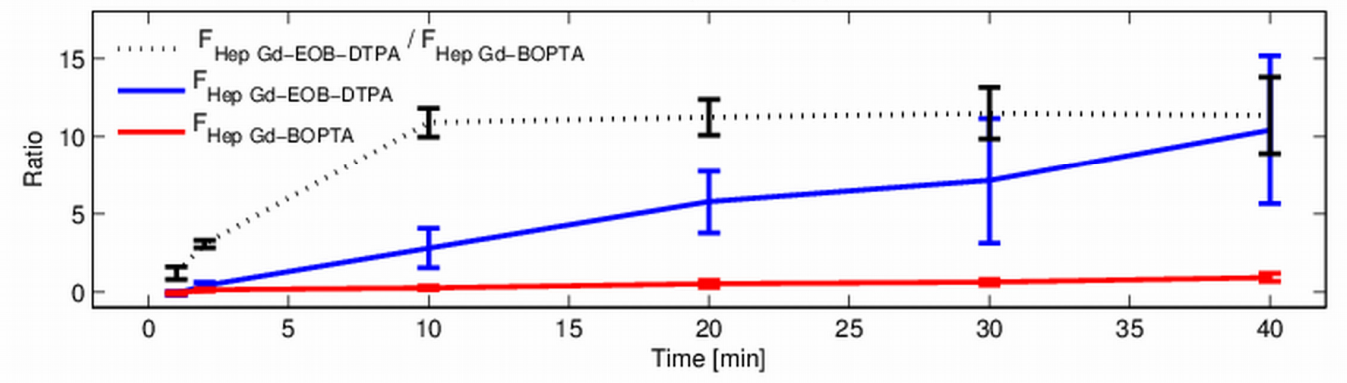

Fig. 4. UPPER PANEL: Signal intensities (SI) measured in liver (solid lines) and spleen (dotted lines) for Gd-EOB-DTPA (blue lines) and Gd-BOPTA (red lines) from 0 to $40 \mathrm{~min}$, for all ten volunteer subjects. UPPER MIDDLE PANEL: Normalised SI $(S$, normalised using the intrinsic, unenhanced SI; Eq. 1). LOWER MIDDLE PANEL: Estimated increase in relaxation rate $\left(\Delta R_{1}\right)$. LOWER PANEL: $F_{\text {Hep }}$ for Gd-EOB-DTPA and Gd-BOPTA and the ratio between these estimates (black dotted line). The curves represent mean value and standard error (SE) for all subjects. 

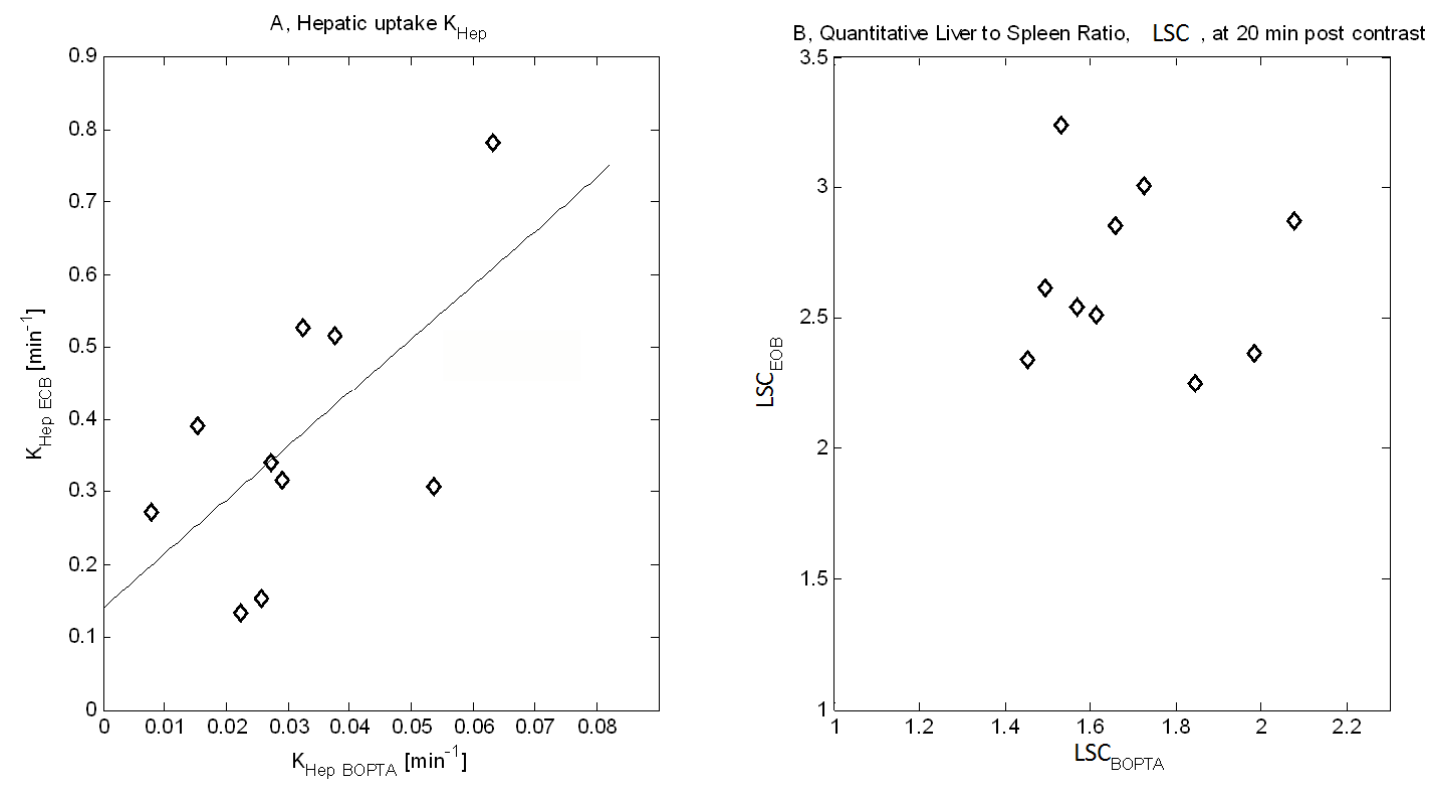

Fig. 5. PANEL A: Correlation plot of the estimated $K_{\text {Hep }}$ for all ten volunteer subjects using GdEOB-DTPA and Gd-BOPTA respectively. The correlation was significant $(p<0.05)$, with $r=0.64$. Fitted regression slope was (estimate \pm standard error) $7.42 \pm 3.66$ and fitted intercept was $0.14 \pm 0.12$. If the intercept was constrained to zero, the estimated slope was $11.0 \pm 1.65$. The results from the preclinical studies on Gd-EOB-DTPA and Gd-BOPTA indicate an expected slope of 10. PANEL B: Correlation plot of the quantitative liver-tospleen contrast ratio, LSC, for the same subjects. No correlation between the contrast agents was observed. 